\title{
Algorithm design and Simulation of optimal maritime search scheme Peng $\mathrm{Qi}^{1, \mathrm{a}}$ \\ ${ }^{1}$ School of North China Electric Power University, Baoding 071003, China. \\ a1819220373@qq.com
}

Keywords: search algorithms, Monte Carlo simulation,“3б” principle.

\begin{abstract}
Aiming to reduce the losses of shipwreck, this paper employs methods in mathematics and simulation fields to build a progressive search model and give out targeted solutions for each stage. After simplifying the actual process of search and rescue, based on Air France Flight 447 (2009) case data, we put forward two kinds of search algorithms to conduct and optimize the maritime search scheme. In order to compare with different sea search arrangements, using Monte Carlo simulation and analyzing the different types of search planes, the best choice was set to get the target. The way to assign different search area resorts to " $3 \sigma$ " principle and then identifying the arrangement which can find the wreckage-site more quickly. Meanwhile, 72-hour "golden window" is also considered. Through taking all these factors into consideration, eventually the best Algorithm is attained for maritime search scheme.
\end{abstract}

\section{Introduction}

A series of major accidents at sea in recent years, such as Malaysia Airlines MH730 event, have resulted in significant loss of life and economy to our community. It urges to pay more and more attention to deal with emergencies at sea. After the shipwreck occurred, in order to reduce casualties and costs, it is very important that whether we can quickly find them and implement the rescue of persons in distress. Sea targets, however, due to the influence of factors such as wind, wave and flow, constantly drift. Especially the shipwreck often occurs under bad weather conditions, which has brought great difficulties to find the search target quickly. It is obvious that maritime search in the whole search and rescue system is the most expensive, dangerous and complex part, so a good scheme is of great significance to improve the success rate of that ${ }^{[1]}$.

In particular, the impact of the vagaries of wind often causes huge impact on the flight. Such as the MH370 and AF447 cases in recent years, it is very often that aircrafts disappeared on specific routes. In order to find the aircraft quickly and give timely rescue at sea, we need to design a offshore optimal search algorithm.

Though sea patrol is difficult to conduct, fortunately it's relatively easy in initial positioning so that we can narrow the search area to a small scale and start the surface search for the missing one. By choosing proper mathematical statistics, algorithm and simulation, we can make full use of the search planes with different equipment and enhance the efficiency of action.

\section{Algorithm design}

\section{Five types of search planes}

Maritime search and rescue are mainly composed of the planes. There are many kinds of cruise machine. To simplify the simulation process, integrated aircraft parameters can be established as follows: 
Table 1 Five types of planes

\begin{tabular}{cccccc}
\hline Types & $\mathrm{A}+$ & $\mathrm{A}$ & $\mathrm{A}-$ & $\mathrm{B}+$ & $\mathrm{B}$ \\
\hline Voyage (n mile) & 4500 & 2100 & 4000 & 3600 & refueled \\
speed(kn/h) & 490 & 330 & 320 & 500 & 150 \\
Accurancy pa & $95 \%$ & $90 \%$ & $85 \%$ & $80 \%$ & $70 \%$ \\
Area (n mile ${ }^{2}$ ) & 2500 & 900 & 1225 & 1225 & 400 \\
\hline
\end{tabular}

\section{Probability Density Distribution}

When a plane is gone on the way to a given point, it proves that the probable density distribution obeys Gaussian distribution. Here Gaussian distribution can be defined in two patterns ${ }^{[2]}$ :one is that, the missing one can be found mostly in the vicinity of its airline, and the farther away from the route, the lower the probability to be found, obeying Gaussian distribution. Based on this distribution, when selecting different types of planes, commanding officers may resort to it for deciding the size of forces to execute tasks and whether the forces be involved in the search operation $^{[3]}$. The other is, the farther away from the last known position, the lower the probability to be found. Also this speculation relays on a Gaussian distribution. The 2 patterns are shown below, where waypoint stands for the nearest site to detect and last-known position indicates the start point to search:

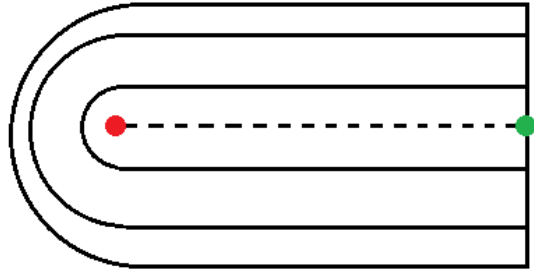

(a)

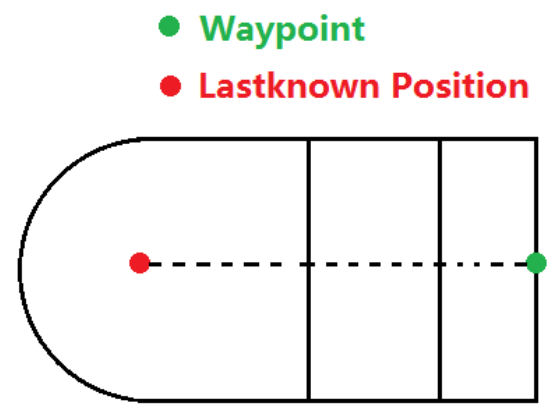

(b)

Figure.1Two patterns of probability density distribution

A comparison is needed to testify which pattern is better. We have set that there are 5 types of search planes involved in the rescue and all of them have different ability for search. When defining the criterion to evaluate our model, we regard the method using less planes to achieve a higher success rate in a short time as the best one. In order to make the results comparable, both the two search areas are divided by the " $3 \sigma$ " principle in Gaussian distribution. Due to most search process on maritime is only by sight, and therefore at this surface search stage just the planes with Grade A-, $\mathrm{B}+$ and $\mathrm{B}$ will shoulder all the tasks to meet the requirements, and at this moment Grade $\mathrm{A}+$ and $\mathrm{A}$ can wait for more urgent undersea rescues.

\section{Search Pattern}

Usually the search scale after location is still too vast to start the search and rescue, under this circumstance it's an effective way to use the parallel sweep search pattern to hunt floating wreckage. This pattern is used to divide a large cross-section into several partitions and assign different search power, enabling them to carry out search operations in coordination. When executing this pattern, search facilities see search corner as the starting point. Diagram is as follows: 


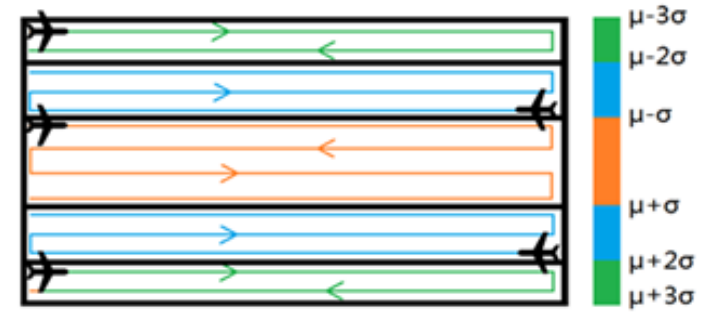

(a)

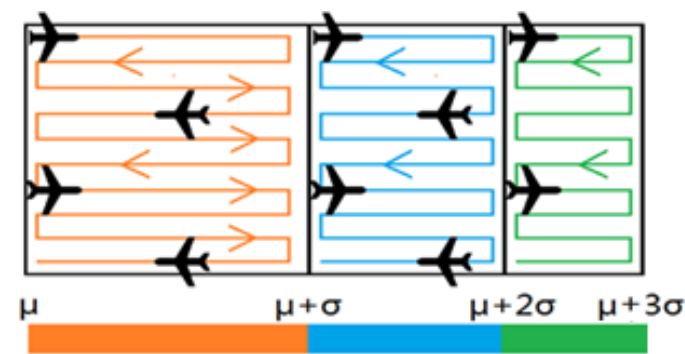

(b)

Figure. 2 Two patterns of search

For both two patterns given above, in the i region, the flight number $\mathrm{n}$ is taken as:

$$
n_{i}=\left\lceil\frac{S_{i}}{S_{a i}}\right\rceil
$$

Where $S_{\mathrm{i}}$ is the area of region $i, S_{\mathrm{ai}}$ is the total area managed by given planes in the $i$ region .

\section{Monte Carlo Simulation}

By using Monte Carlo simulation ${ }^{[4]}$, the two patterns can be compared according to the steps below:

(1) Generate n numbers of points as wreckage in the given region at random.

(2) Departure from the corner of jurisdiction, each aircraft search its region by parallel sweep search pattern.

(3) In the simulation process, when a plane comes from the hit point, whether it can find the wreckage rely on its accuracy.

(4) If not succeed at one time, the plane goes backtrack to start searching again, until having found all the points, then record the total time it wasted in this task.

\section{Results}

Now we use case AF447 as data to compare the two patterns, the randomly generated number is set to 8 , after 300 times of naval search simulation, and take the average time, the results is shown as follows:

Table 2. The results of simulation in the surface search model

\begin{tabular}{cccccc}
\hline Pattern & $\begin{array}{c}\text { Search area } \\
(\mathrm{n} \mathrm{mile})\end{array}$ & $\begin{array}{c}\text { Number } \\
\text { of A- }\end{array}$ & $\begin{array}{c}\text { Number } \\
\text { of B+ }\end{array}$ & $\begin{array}{c}\text { Number } \\
\text { of B }\end{array}$ & $\begin{array}{c}\text { Total time } \\
\text { (h) }\end{array}$ \\
\hline 1 & \multirow{2}{*}{$48.74 \times 114.05$} & 4 & 2 & 2 & 104.67 \\
2 & 4 & 2 & 2 & 152.5 \\
\hline
\end{tabular}

\section{Summary}

Conclusions can be obtained as follows:

(1) When applying to Gaussian distribution to partition the ocean, each search pattern is allocated equal planes, so we can think of using this way to divide the given area is fair.

(2) With the same simulation conditions, pattern 1 uses less time than pattern 2. That is, in the implementation of maritime search and rescue, pattern 1 has advantages on a faster time to find more wreckage.

Also to be noted is that we define the initial 72 hours after the accident occurred as 72-hour "golden window", during which period passengers' survival probability is high, so at this time 
rescue is the most important thing. In the actual course of search and rescue, maritime search time should be controlled in this range and it can be considered that over this time, the collected information is enough to determine the position of wreckage fell into the sea, and then start the further search.

\section{Reference}

[1] Li Jie. Design and Application of Aided Decision System for Salvage and Rescue at Sea. (2011)

[2] Raj Chhikara. The Inverse Gaussian Distribution: Theory: Methodology, and Applications. (1988)

[3] Xing Shengwei.Model and Simulation of three-dimensional global maritime search optimization[D].Dalian Maritime University, 2012.

[4] G.S. Fishman.Monte Carlo: Concepts, Algorithms, and Applications, volume 1 of Springer Series in Operations Research. Springer-Verlag, New York, 1996. 